\title{
Effects of Light-Emitting Diode Light on Human Electroencephalogram in Comparison with Fluorescent Light
}

\author{
Gwan-Taek Lee ${ }^{1}$, Chany Lee ${ }^{1}$, Daeyoung Kim² ${ }^{2}$ HyunTaek Kim³ ${ }^{3}$ SungHo Woo ${ }^{3}$ and Ki-Young Jung ${ }^{1}$ \\ ${ }^{1}$ Department of Neurology, Korea University Medical Center, Korea University College of Medicine, Seoul, \\ ${ }^{2}$ Department of Neurology, Samsung Seoul Hospital, Seoul, \\ ${ }^{3}$ Department of Psychology, College of Liberal Arts, Korea University, Seoul, Korea
}

Received November 21, 2012 Revised December 19, 2012 Accepted December 19, 2012

Address for correspondence Ki-Young Jung, MD Department of Neurology,

Korea University Medical Center, Korea University

College of Medicine,

73 Inchon-ro, Seongbuk-gu,

Seoul 136-705, Korea

Tel: +82-2-920-6649

Fax: +82-2-925-2472

E-mail: jungky@korea.ac.kr
Objectives: Selecting suitable illumination is an integral part of increasing productivity in the office or factory, because poor lighting conditions may often cause decreased work efficiency. Light emitting diode (LED) light is becoming recognized as one of the most promising general sources of illumination. We conducted spectral power analysis of electroencephalograms (EEGs) obtained during resting and cognitive task activities to identify the effects on human arousal and cognitive performance under LED light compared with conventional fluorescent light. Methods: Thirteen healthy, right-handed students participated in the present study. Each subject took part in two experimental sessions, one under fluorescent and one under LED lighting conditions. The experimental measurements consisted of a resting state EEG, an event-related potential (ERP) during a visual working memory (VWM) task, and a questionnaire about subjective feelings regarding the lighting conditions. The EEG power spectra, the amplitude and latency of the P300 ERP component, the behavioral responses for the VWM task, and the questionnaire data were compared for the two lighting conditions. Results: The EEG spectral power showed no difference between the LED and fluorescent lighting conditions. The amplitude of the P300 component decreased significantly with increasing numbers of items, while there were no differences between the two lighting conditions. Additionally, behavioral responses and subjective feelings were the same under the two lighting conditions. Conclusions: Our study suggests that there are no significant differences between LED light and fluorescent light on the human arousal state and VWM.

J Korean Sleep Res Soc 2012;9:28-33

\section{Introduction}

We cannot imagine any working environment without illumination in modern society, because humans greatly rely on optical information for their productivity. Workplaces with poor lighting conditions often cause laborers to be stressed or even to have serious work accidents. This occurs because light exerts not only visual effects but also nonvisual effects on numerous physiological variables, such as the human sleep-wake cycle and cognitive performance, primarily through properties such as dose, duration, timing, and wavelength. ${ }^{1}$ Thus, selecting suitable illumination is an integral part of increasing productivity in the office or factory.

Although fluorescent lamps are still the dominant source of artificial illumination in modern living environments, light emitting diodes (LEDs) have begun to attract interest as one of the most promising candidates for sources of general illumin- ation in the near future. In fact, according to a market research report from BCC Research, the global market for LED lights noticeably increased by more than $85 \%$ during the period 2005-2009. ${ }^{2}$ LEDs provide technical advantages over fluorescent lamps, such as lower power consumption, longer lifetime, greater eco-friendliness due to not using mercury, and easier processing due to their small size and adaptable shape. ${ }^{3}$

It is well known that lighting can cause acute emotional, behavioral, and cognitive changes. ${ }^{4-6}$ Additionally, a large body of research shows the chronic effects of lighting on sleep and neurocognitive function. ${ }^{1,78}$ These effects of light may depend on parameters such as intensity, wavelength, and source of light. Thus, prior to a shift to new a lighting source (i.e., LED light) in artificial luminous environments, issues of whether it might affect human physiology or cognition should be considered to ensure that workplace efficiency are maintained. To our knowledge, only one study has reported on this issue, finding that 
LED light had positive effects on worker behavioral performance during several cognitive tasks, compared with traditional fluorescent lamps. ${ }^{9}$ However, since the study did not evaluate the concurrent electrophysiological responses of brain activity, information processing at a cortical level was not demonstrated.

Electroencephalography (EEG) provide a sensitive means of measuring psychological as well as physiological human states. The resting state EEG reflects a particularly important state of arousal, which can be characterized using frequency analysis. In addition, event-related potentials (ERPs) provide a neurophysiological index of a subject's cognitive functioning. Because the lighting condition exerts a direct influence on visual function, the ERP paradigm using visual stimuli is used to investigate the lights' effects. Specifically, ERP measurements, such as examining the $\mathrm{P} 300$ and behavioral responses during the visual working memory (VWM) task, allow objective assessments of cognition and behavior through visual functioning. Thus, the study of EEGs and ERPs in combination appears to be useful for exploring human neurophysiology reflecting arousal states, cognitions, and task performance. Nevertheless, no study has yet addressed the electrophysiological differences between LED and fluorescent light.

In the present study, to identify the effects of LED light on EEGs during rest and during performance of a cognitive task, compared with conventional fluorescent light, we analyzed the spectral power of EEGs obtained during resting states and ERP during VWM tasks.

\section{Methods}

\section{Setting}

The experiments took place in a laboratory with the window completely covered with black curtains to prevent outside light from entering the room. Four tube-straight threeband fluorescent lamps (FLU) and four similarly shaped binary-complementary white LED lamps were alternately placed two meters above the floor. The spectra irradiance of each light is shown in Fig. 1. Each subject underwent the experimental procedures while sitting in a comfortable chair about one meter from the installed lighting.

\section{Experimental procedure}

Thirteen healthy, right-handed university students (six males, 7 females; aged $23.3 \pm 1.6$ years) participated in this study. Each subject gave written informed consent to participate. The experimental protocol was approved by the Institutional Review Board of Korea University Medical Center. Participants visited our laboratory at 19:00. Each subject performed two experimental sessions, one under each lighting condition:

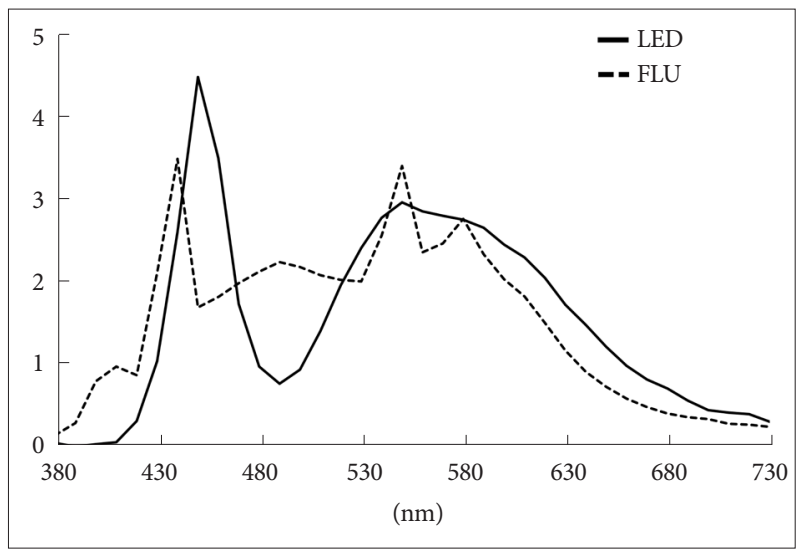

Fig. 1. The spectra irradiances of the LED (solid line) and the fluorescent tube (dotted line). LED: light emitting diode, FLU: fluorescent lamp.

fluorescent and LED. One session consisted of spontaneous EEG for 10 minutes, ERP during a VWM task for 15 minutes, and a questionnaire to obtain the visual analogue score (VAS). A rest time of 5 minutes was given between the two sessions. At the beginning of each session, the illuminance was adjusted so that the two lighting conditions were matched, and then color temperature was measured. The order of fluorescent and LED sessions was counterbalanced among the participants to control the order effect.

\section{The resting state EEG}

The EEGs were recorded using a 64-channel digital EEG machine (Grass Neurodata Acquisition System, Grass Technologies, Quincy, MA, USA) with a cap electrode (Quick-Cap, Compumedics Neuroscan, Charlotte, NC, USA). The reference electrode was set to linked earlobes, impedance was kept below $10 \mathrm{k} \Omega$, and the band-pass filter setting was $0.3-70 \mathrm{~Hz}$ with a sampling rate of $1600 \mathrm{~Hz}$. Two electrooculography channels (placed on the left and right outer canthi) were added to confirm eyeball movements.

The resting state EEG of each subject was reviewed for offline analysis, and 10 artifact-free 2-s epochs in the eye-open state were selected per subject. Each epoch was transformed into the frequency domain using Fast Fourier Transforms, and then the power spectral density function for each subject was evaluated. Absolute power for the five frequency bands (delta: 1-3 Hz, theta: $4-7 \mathrm{~Hz}$, alpha: $8-12 \mathrm{~Hz}$, beta: $13-30 \mathrm{~Hz}$, and gamma: $30-45 \mathrm{~Hz}$ ) was determined at $\mathrm{Fz}, \mathrm{Cz}$, and $\mathrm{Pz}$ electrodes.

\section{Visual working memory task}

A VWM task was used to estimate participants' behavioral and cognitive responses under each lighting condition. All stimuli were presented on a 17 -inch LCD monitor with a grey background using commercial software (PRESENTATION; Neurobehavioral systems, Berkeley, CA, USA). Each partici- 
pant underwent 300 trials in each condition. The test array was shown for $2 \mathrm{~s}$, at $1 \mathrm{~s}$ after the memory array onset in each trial. Participants were instructed to indicate whether or not two arrays were identical by pushing one of two buttons as quickly as possible after they were presented with the test array. A stimulus array consisted of $1,2,3,4,8$ or 12 items that were colored squares. The color of each item was randomly selected from red, blue, violet, green, yellow, black, or white. Item positions were also randomized in each trial. The color of only one item in the test array was different from the corresponding item in the memory array in $50 \%$ of the trials. They were identical in the remaining trials.

Event-related potential epochs were extracted at between -200 and $+1200 \mathrm{~ms}$ after the memory array onset and saved for later off-line analysis. Baselines were corrected by subtracting the root mean square of the pre-stimulus interval from the whole epoch lengths. Only those trials with correct responses were included in the ERP analysis. Independent component analysis was applied to correct stereotyped ocular and muscular artifacts (Jung et al. 2000). ERP epochs were averaged for different numbers of items in the memory array separately. ERP latencies and amplitudes were measured relative to their pre-stimulus baseline. The P300 component was defined as points with positive peak amplitudes between 300 and 500 $\mathrm{ms}$ at the Pz recording site. The amplitudes of the $\mathrm{P} 300$ components were averaged over the $\pm 25 \mathrm{~ms}$ time window relative to the latency of each peak.

\section{Questionnaire}

Subjective brightness, visual fatigue, and satisfaction (from 0 to 10 points) was checked as visual analogue scale (VAS) in the questionnaire for subjects to assess each lighting condition immediately after the ERP tasks.

\section{Statistical analysis}

Electroencephalograms, ERP, and behavioral responses were analyzed by repeated measures analysis of variance (ANOVA). The within-subject variables of the spectral power for each frequency band (delta, theta, alpha, beta, and gamma) of the resting state EEG were channel (three levels: $\mathrm{Fz}, \mathrm{Cz}$, and $\mathrm{Pz}$ ) and light (two levels: fluorescent and LED). The amplitude and latency of the P300 component during VWM tasking were each analyzed at the $\mathrm{Pz}$ recording site; the within-subject variables were number of items (six levels: 1, 2, 3, 4, 8, and 12) and light (two levels: fluorescent and LED). For the hit rate and the reaction time of behavioral responses, the withinsubject variables were identical to those for the $\mathrm{P} 300$ analysis. The Greenhouse-Geisser correction was used to evaluate F ratios in order to control for Type 1 error in the repeated measures design. Bonferroni post hoc tests were used to identify the sources of significant variance. The questionnaire results were analyzed using paired t-tests for comparisons with unequal variance for the fluorescent and LED lights. The dependent variables were the VAS scores of brightness, visual fatigue, and satisfaction based on the questionnaires. Statistical significance was defined as $p$ values of $<0.05$.

\section{Results}

The fluorescent and LED lighting had color temperatures of 8690 and $6660 \mathrm{~K}$, respectively. Light intensities were matched, with 340 lux in both conditions.

\section{EEG power spectra}

Electroencephalograms spectral powers for each frequency band at the three midline electrodes are shown in Fig. 2. For each frequency band, ANOVA revealed that light showed no
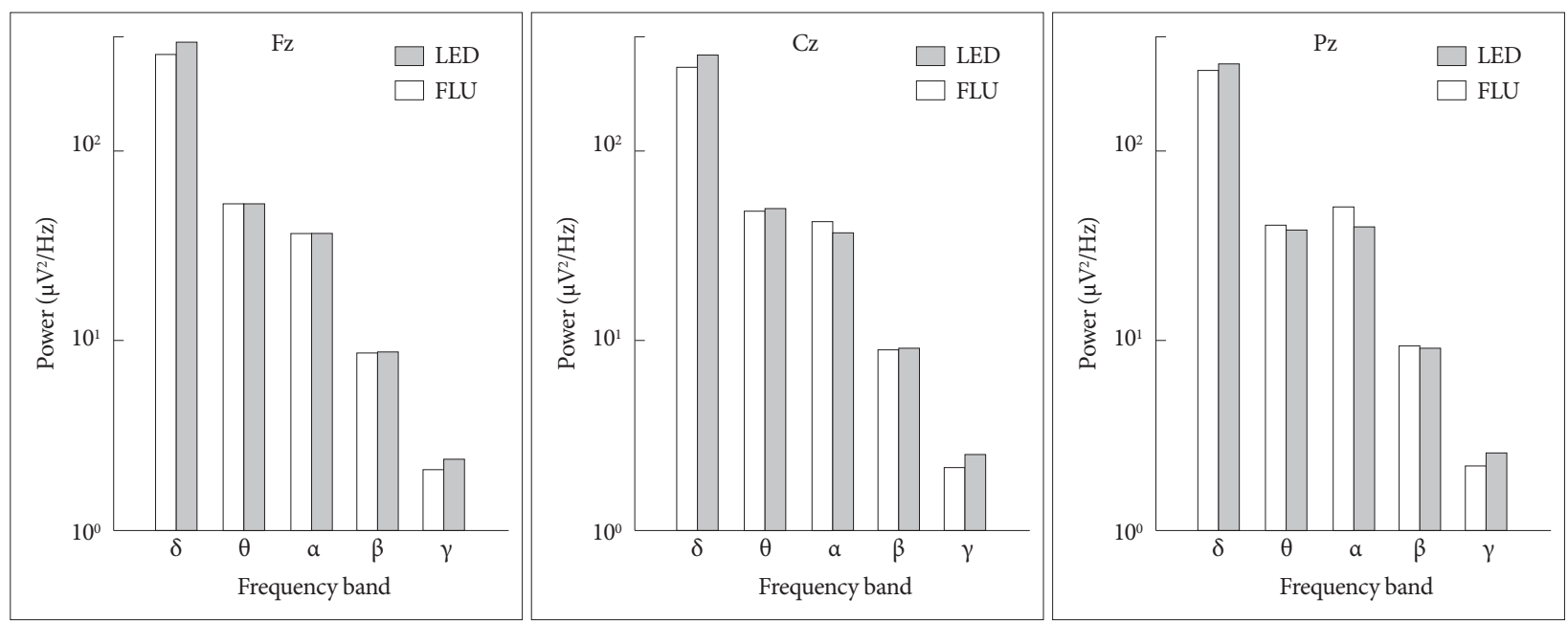

Fig. 2. Mean electroencephalogram spectral power within each frequency band for all subjects at $\mathrm{Fz}, \mathrm{Cz}$, and $\mathrm{Pz}$ electrodes $(\delta: 1-3 \mathrm{~Hz}, \theta$ : 4-7 Hz, $\alpha: 8-12 \mathrm{~Hz}, \beta: 13-30 \mathrm{~Hz}, \gamma: 30-45 \mathrm{~Hz})$. 
Table 1. Summary of analysis of variance of electroencephalogram power spectra

\begin{tabular}{|c|c|c|c|c|c|c|}
\hline \multirow{2}{*}{ Factor } & \multicolumn{2}{|c|}{ Channel } & \multicolumn{2}{|c|}{ Light } & \multicolumn{2}{|c|}{ Channel $\times$ Light } \\
\hline & $\mathrm{F}$ & $\mathrm{p}$ & $\mathrm{F}$ & $\mathrm{p}$ & $\mathrm{F}$ & $\mathrm{p}$ \\
\hline Delta & 10.662 & 0.006 & 2.121 & NS & 0.328 & NS \\
\hline Theta & 9.706 & 0.003 & 1.011 & NS & 6.485 & 0.020 \\
\hline Alpha & 10.751 & 0.001 & 0.031 & NS & 3.065 & NS \\
\hline Beta & 3.356 & 0.087 & 0.016 & NS & 1.216 & NS \\
\hline Gamma & 3.352 & NS & 0.853 & NS & 0.633 & NS \\
\hline
\end{tabular}

NS: not significant
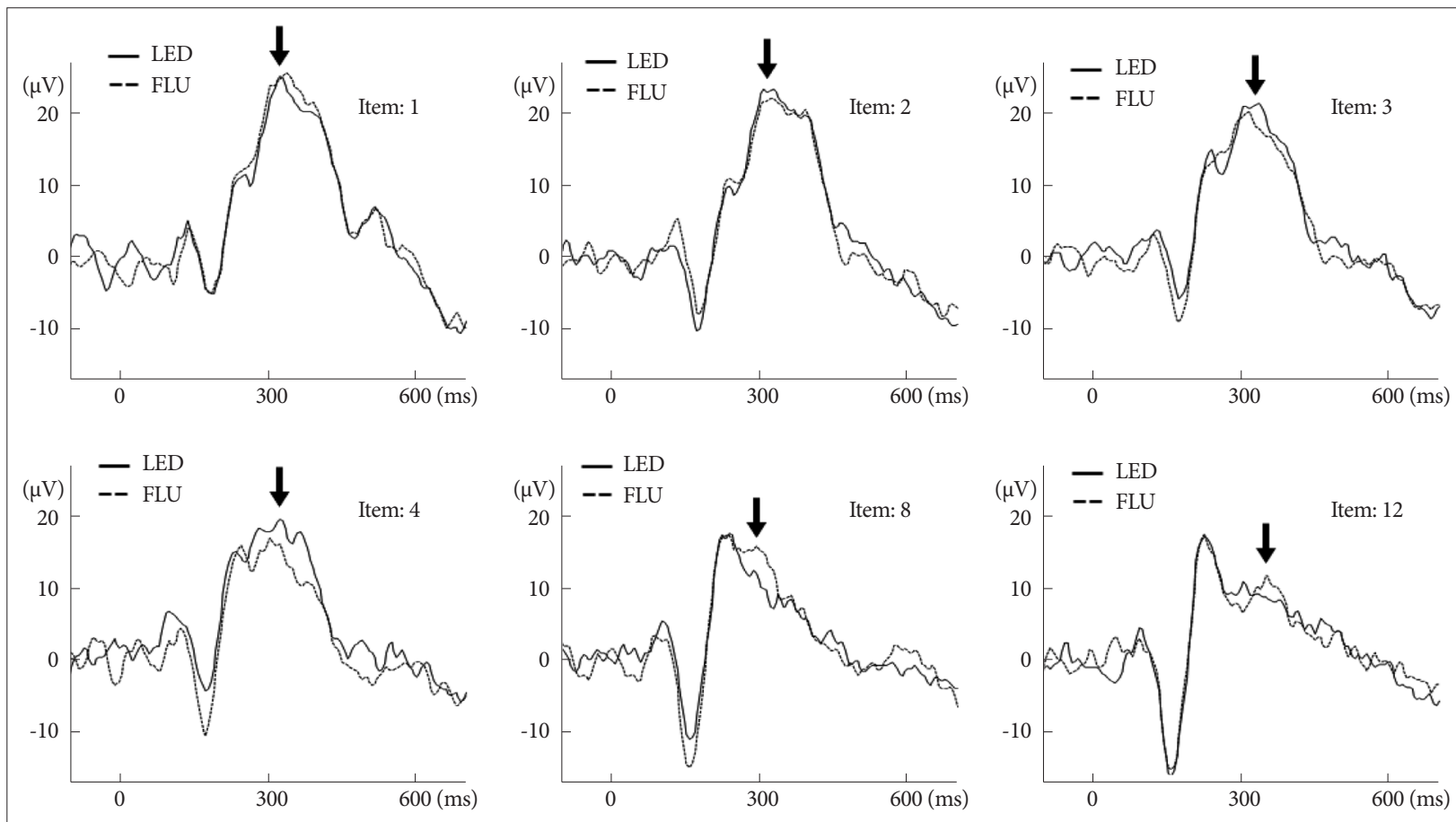

Fig. 3. Grand averaged ERP waveforms at Pz electrode. Each panel corresponds to the number of items during the visual working memory task, from one (top left) to twelve (bottom right). Arrows indicate P300 ERP components. FLU: fluorescent lamp, LED: light-emitting diode, ERP: event-related potential.

significant main effect, although significant main effect was observed with channel. The interaction between channel and light was not significant (Table 1).

\section{ERP analysis}

Event-related potential waveforms at the Pz electrode site are shown in Fig. 3. The P300 components were identified approximately $350 \mathrm{~ms}$ after the memory array onset. The number of items showed a significant main effect on the P300 amplitudes $\left(\mathrm{F}_{2.11,23.26}=9.97, \mathrm{p}=0.001\right)$, while light $\operatorname{did} \operatorname{not}\left(\mathrm{F}_{1.0,11.0}=\right.$ $0.04, p>0.05$ ), and the interaction between the number of items and the light did not $\left(\mathrm{F}_{3.1734 .92}=1.45, \mathrm{p}>0.05\right)$. The number of items $\left(\mathrm{F}_{3.39,37.33}=1.32, \mathrm{p}>0.05\right)$, the light $\left(\mathrm{F}_{1.0,11.0}=3.1, \mathrm{p}>\right.$ $0.05)$, and the interaction of number of items and light $\left(\mathrm{F}_{3.35}\right.$, $36.83=0.59, \mathrm{p}>0.05$ ) showed no significanteffects on latency.

\section{Behavioral responses}

Fig. 4. shows the change in behavioral responses as the number of items increased, with a significant main effect on hit rate $\left(\mathrm{F}_{2.62,28.83}=199.17, \mathrm{p}<0.001\right)$ and reaction time $\left(\mathrm{F}_{1.8,19.8=}\right.$ $\left.{ }_{24.73}, \mathrm{p}<0.001\right)$. The light showed neither significant main effect on hit rate $\left(\mathrm{F}_{1.0,11.0=3.82}, \mathrm{p}>0.05\right)$ nor reaction time $\left(\mathrm{F}_{1.0,11.0=}\right.$ $0.02, \mathrm{p}>0.05)$, and the interaction between light and number of items also showed no significant effect on behavioral responses (hit rate: $\mathrm{F}_{2.44,26.83}=1.52$, $\mathrm{p}>0.05$; reaction time: $\mathrm{F}_{2.08,22.85}=$ $1.09, \mathrm{p}>0.05)$.

\section{Questionnaire}

The results of the subjective assessment of lighting conditions are shown in Table 2. Pairwise comparisons of the VAS scores for brightness, visual fatigue, and satisfaction showed no significant differences between LED and fluorescent light- 

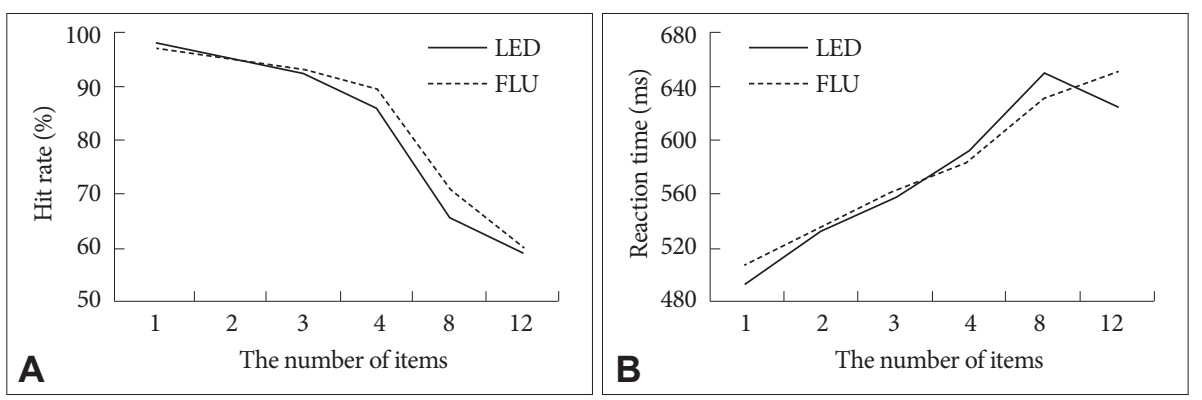

Fig. 4. Hit rate $(A)$ and reaction time (B) with respect to the number of items during the visual working memory task.
Table 2. The results of the subjective assessment of lighting conditions

\begin{tabular}{cccc}
\hline Group & LED & FLU & p \\
\hline Brightness & $6.21 \pm 1.86$ & $6.01 \pm 1.46$ & NS \\
Visual fatigue & $4.87 \pm 2.42$ & $4.51 \pm 2.63$ & NS \\
Satisfaction & $5.63 \pm 1.89$ & $5.34 \pm 1.96$ & NS \\
\hline
\end{tabular}

LED: light emitting diode, FLU: fluorescent lamp, NS: not significant

ing conditions.

\section{Discussion}

In this study, we analyzed the EEG spectral power measured during a resting state, ERP components elicited by a VWM task, and subjective data obtained with a questionnaire in order to compare the effects of LED and fluorescent light on human cognitions, behaviors, and subjective feelings. In our study, the intensity of light was matched between two lighting conditions. Although the color temperature could not be matched perfectly due to original differences in the spectral irradiance of the two light sources, this is unlikely to affect the results because the spectral composition has no effect on the performance of simple cognitive tasks. ${ }^{10)}$

The EEG reflects a particularly important physiological state of arousal which can be characterized using frequency analysis. ${ }^{11)}$ In particular, alpha oscillation is a sensitive measure of attentional demands in cognitive tasks, ${ }^{12)}$ and further, fluorescent light with low frequency ballasts has significantly decreased alpha power compared with high frequency ballasts. ${ }^{6}$ Nevertheless, we fund no significant differences in EEG spectral power along all frequency bands, between the LED and fluorescent light conditions in our study. This indicates that LED lighting does not disturb human attention, compared with conventional lighting.

The amplitude and latency of the P300 are sensitive to task processing demands and the time required for detecting and evaluating a stimulus. These indexes vary with individual differences in cognitive capability. ${ }^{13)}$ Our findings indicate that increasing the number of items significantly decreases P300 amplitude; however, the lighting condition did not affect P300 amplitude or latency. Similarly, the hit rate and reaction time were not changed by the lighting condition, but they were affected by the task difficulty. In addition, VAS scores for the two lighting conditions were not significantly different, which means that the subjects reported no noticeable differences in subjective feelings regarding brightness, visual fatigue, and satisfaction between fluorescent and LED lighting.

Hawes et al.9. recently reported that LED lighting may have positive implications for work performance, compared to traditional fluorescent lighting. However, this finding could lead to misunderstanding because the fluorescent lighting used in their experiment had lower luminance and color temperature than the LED lighting. In contrast, our results indicate that the LED lighting does not significantly effect on cognitive and behavioral performance. In conclusion, our findings suggest that there are no differences in the effects on the human arousal state or cognitive performance between the lighting conditions provided by LED and fluorescent light sources. As we investigated only short-term effect with relatively small sample, long-term effect of LED light should be studied further in the future.

\section{Acknowledgments}

This work was supported by Industrial Strategic Technology Development Program (Project No: 10037416, Establishment of infrastructure for LED-marine convergence technology support and technology development for commercialization) funded by the Ministry of Knowledge Economy (MKE, Korea).

\section{REFERENCES}

1. Chellappa SL, Gordijn MCM, Cajochen C. Chapter 7 - Can light make us bright? Effects of light on cognition and sleep. In: Van Dongen HPA, Kerkhof GA. Progress in Brain Research. Human Sleep and Cognition Part II: Clinical and Applied Research. Vol 190. New York: Elsevier, 2011; 119-133.

2. Eckard R. Energy-efficient Technologies: The Global Market. 2009.

3. Mehta R, Deshpande D, Kulkarni K, Sharma S, Divan D. LEDs - A Competitive Solution for General Lighting Applications. Energy 2030 conference. ENERGY 2008. IEEE, 2008;1-5.

4. Vandewalle G, Schwartz S, Grandjean D, et al. Spectral quality of light modulates emotional brain responses in humans. Proc Natl Acad Sci U S A 2010;107:19549-19554.

5. van Bommel WJ. Non-visual biological effect of lighting and the prac- 
tical meaning for lighting for work. Appl Ergon 2006;37:461-466.

6. Küller R, Laike T. The impact of flicker from fluorescent lighting on well-being, performance and physiological arousal. Ergonomics 1998; 41:433-447.

7. Gooley JJ, Chamberlain K, Smith KA, et al. Exposure to room light before bedtime suppresses melatonin onset and shortens melatonin duration in humans. J Clin Endocrinol Metab 2011;96:E463-E472.

8. Cajochen C, Zeitzer JM, Czeisler CA, Dijk DJ. Dose-response relationship for light intensity and ocular and electroencephalographic correlates of human alertness. Behav Brain Res 2000;115:75-83.

9. Hawas BK, Brunyé TT, Mahoney CR, Sullivan JM, Aall CD. Effects of four workplace lighting technologies on perception, cognition and affective state. International Journal of Industrial Ergonomics 2012;42:
122-128.

10. Boray PF, Gifford R, Rosenblood L. Effects of warm white, cool white and full-spectrum fluorescent lighting on simple cognitive performance, mood and ratings of others. Journal of Environmental Psychology 1989;9:297-307.

11. Jung KY, Koo YS, Kim BJ, et al. Electrophysiologic disturbances during daytime in patients with restless legs syndrome: further evidence of cognitive dysfunction? Sleep Med 2011;12:416-421.

12. Ward LM. Synchronous neural oscillations and cognitive processes. Trends Cogn Sci 2003; 7:553-559.

13. Polich J. Updating P300: an integrative theory of P3a and P3b. Clin Neurophysiol 2007;118:2128-2148. 